\title{
Intelligent Traffic Information System Based on Integration of Internet of Things and Agent Technology
}

\author{
Hasan Omar Al-Sakran \\ Management Information Systems Department \\ King Saud University \\ Riyadh, Saudi Arabia
}

\begin{abstract}
In recent years popularity of private cars is getting urban traffic more and more crowded. As result traffic is becoming one of important problems in big cities in all over the world. Some of the traffic concerns are congestions and accidents which have caused a huge waste of time, property damage and environmental pollution. This research paper presents a novel intelligent traffic administration system, based on Internet of Things, which is featured by low cost, high scalability, high compatibility, easy to upgrade, to replace traditional traffic management system and the proposed system can improve road traffic tremendously. The Internet of Things is based on the Internet, network wireless sensing and detection technologies to realize the intelligent recognition on the tagged traffic object, tracking, monitoring, managing and processed automatically. The paper proposes an architecture that integrates internet of things with agent technology into a single platform where the agent technology handles effective communication and interfaces among a large number of heterogeneous highly distributed, and decentralized devices within the IoT. The architecture introduces the use of an active radio-frequency identification (RFID), wireless sensor technologies, object ad-hoc networking, and Internet-based information systems in which tagged traffic objects can be automatically represented, tracked, and queried over a network. This research presents an overview of a framework distributed traffic simulation model within NetLogo, an agentbased environment, for IoT traffic monitoring system using mobile agent technology.
\end{abstract}

Keywords-Intelligent Traffic; Internet-of-Things; RFID; Wireless Sensor Networks; Agent Technology

\section{INTRODUCTION}

In recent years popularity of private motor vehicles is getting urban traffic more and more crowded. As result traffic monitoring is becoming one of important problems in big smart-city infrastructure all over the world. Some of these concerns are traffic congestion and accidents that usually cause a significant waste of time, property damage and environmental pollution. Any type of congestion on roads ultimately leads to financial losses. Therefore, there is an urgent need to improve traffic management. The appearance of the Internet of Things (IoT) provides a new trend for intelligent traffic development.

This research proposes to employ the IoT, agent and other technologies to improve traffic conditions and relieve the traffic pressure. Information generated by traffic IoT and collected on all roads can be presented to travelers and other users. Through collected real-time traffic data, the system can recognize current traffic operation, traffic flow conditions and can predict the future traffic flow. The system may issue some latest real-time traffic information that helps drivers choosing optimal routes. Therefore, the system can precisely administrate, monitor and control moving vehicles. Constructing an intelligent traffic system based on IoT has a number of benefits such improvement of traffic conditions, reduction the traffic jam and management costs, high reliability, traffic safety and independence of weather conditions $[1,2]$.

Such traffic IoT must include every element of traffic such as roads, bridges, tunnels, traffic signals, vehicles, and even drivers. All these items will be connected to the internet for convenient identification and management through sensor devices, such as RFID devices, infrared sensors, global positioning systems, laser scanners, etc.

Traffic IoT provides traffic information collection and integration, supporting processing and analysis of all categories of traffic information on roads in a large area automatically and intelligently. Thus, modern traffic management is evolving into an intelligent transport system based on IoT.

Traffic requires suitable information about services and logistics available on the road and therefore the system can become more self-reliable and intelligent. With a number of WSN and Sensor enabled communications, an IoT of data traffic will be generated. This traffic monitoring applications need to be protected to prevent any security attack frequent in urban cities. Few such prototypes implementations can be found in [3, 4] and the Smart Santander EU project [5].

The aim of this paper is to present a framework for realtime traffic information acquisition and monitoring architecture based on the IoT utilizing wireless communications. The primary characteristic of the proposed traffic information infrastructure is its capability of integrating different technologies with the existing communication infrastructures. The proposed architecture allows gathering real-time traffic data generated by sensory units and monitoring the traffic flow using multi-agent based system. Agents can perform specific tasks with a degree of intelligence and autonomy, and interact with their environment in a useful way without human intervention thus decreasing network load, facilitating heterogeneous IoT devices, providing support for collaboration and interoperability in IoT and programmable RFID and WSN, 
overcoming network latency, and asynchronous and autonomous execution.

The remainder of the paper is organized as follows. Background on IoT is discussed in section 2. Section 3 presents related work. Framework structure of the proposed traffic system is introduced in section 4 . Section 5 describes the agent-based approach for the development of intelligent traffic information system. Discussion of the proposed traffic simulation framework is presented in section 6. Finally, section 7 is devoted to conclusions and future work.

\section{INTERNET OF THINGS}

During past few years recent communication paradigm the internet of things - has gained significant attention in academia as well as in industry because it represents an enormous opportunity for cost savings and new revenue generation across a wide range of industries. The main reasons behind this interest are its capabilities. IoT can be used to create a world where all smart objects of our everyday life are connected to the Internet and interact with each other with minimum human involvement to reach a common goal [8]. The term Internet of Things was first appeared by Kevin Ashton [9] in the context of supply chain management.

Gartner forecasts that the IoT will reach 26 billion units by 2020, up from 900 million just five years ago, and this will impact the information available to supply chain leaders. According to Cisco's study, cities all over the world are to claim $\$ 1.9$ trillion in value from IoT over the next decade by building smarter cities based on smarter infrastructure, through providing optimal traffic management, parking, and transit services [10].

The enabling technologies that are expected to form the building blocks of the sensing and communication technologies in IoT are Wireless Sensor Networks (WSN) and RFID-based networks connected together through the Internet or other technologies and protocols. RFID is considered as one of the leading technologies mainly due to its low cost, and its strong support from the business community. RFID can transform everyday objects into smart objects. Sensor network integrates different technologies, such as sensor, distributed information processing, embedded computing and wireless communications. Sensors and RFID are playing a significant role in constructing IoT. Multiple RFID and sensors with computing and communication power are connected into wireless networks and cooperate with each other to exchange collected data with the physical world to accomplish specific tasks.

Implementation of IoT relies on the integration of RFID systems, WSNs, and intelligent technologies. RFID and wireless data communication technology are used to construct a network which covers everything. Objects such as RFID tags and readers, sensors, actuators, mobile phones, smart devices, embedded computers, etc., will be included into the network and will interact with each other through unique addressing schemes [11]. These objects have actuating, processing, storing and networking capabilities. With the advances in sensor technology, sensors will be embedded within all the objects around us. The result will be the generation of huge amounts of data which will have to be stored, processed and presented in efficient and easily interpretable form. IoT allows people and various objects to be connected anytime and anywhere with anything and to any service, and use any network; and communicate with each other in real time as long as they are online $[12,13]$.

Other necessary components include cloud, data modeling, storing, processing, and communication technologies [14]. The major wireless technologies used to build wireless sensor networks are wireless personal area network (Bluetooth), wireless local area network (Wi-Fi), wireless metropolitan area network (WiMAX), wireless wide area network (3G/4G mobile networks) and satellite network (GPS). A typical structure of a RFID based sensor network is presented in fig. 1. It consists of wireless low-end RFID sensor nodes that generate data (tags) and high-end RFID sensor nodes that retrieving data from the low nodes. Data collected by the high nodes are sent to mobile static nodes (readers). Readers send the data to wireless low-end computational devices (base stations). These devices perform a certain amount of processing on the sensor data. Then data sent to high-end computational servers through the internet (or other network) to be processed further and there data will be shared and stored.

\section{RELATED WORK}

A number of researchers have dealt with the problem of intelligent traffic monitoring and controlling, and as a result of their efforts several different approaches have been developed. Pang et al. [15] proposed a traffic flow prediction mechanism based on a fuzzy neural network model in chaotic traffic flow time series. Bhadra et al. [16] applied agent-based fuzzy logic technology for traffic control situations involving multiple approaches and vehicle movements. In [17] the authors developed strategies to integrate different dynamic data into Intelligent Transportation Systems. Patrik et al. [18] proposed a service-oriented architecture (SOA) for an effective integration of IoT in enterprise services.

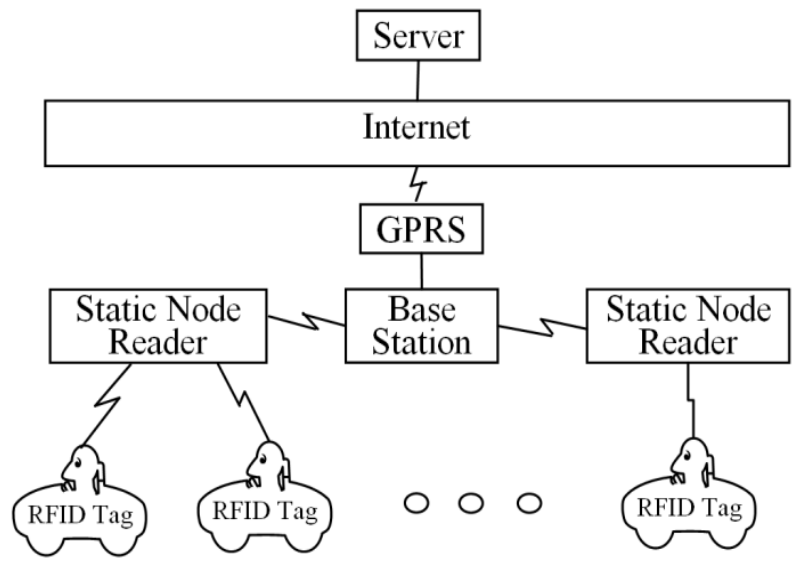

Fig. 1. RFID-based sensor network

Recently researchers shifted their attention to revolutionizing paradigm of the Internet of Things, which resulted in constructing of a more convenient environment composed of various intelligent systems in different domains such as intelligence business inventories, health care, 
intelligent home, smart environment, smart metering, supply chain logistics, retail, smart agriculture, monitoring electrical equipment, etc. [19-22], while it is still in the early stage in case of intelligent transportation system with respect to their needs [23-26]. Different IoT systems such as UbiComp [27], FeDNet $[28,29]$ are using message simple passing techniques for communication. Such techniques consume a large amount of bandwidth and energy.

Agent technology has been implemented in different aspects of the traffic systems such as handling traffic congestion by monitoring the current traffic congestion and providing the optimal route for a vehicle [30-32]. Fortino et al. [33] proposed an architecture integrating agents and cloud computing to develop decentralized smart objects within IoT, while Godfrey et al. [34] used mobile agent to handle not just the communications among devices within the IoT but to conduct searching for needed resources.

\section{FRAMEWORK STRUCTURE OF PROPOSED SYSTEM}

The major tasks of the proposed system are detecting mobile objects and their location, identifying mobile objects and transmitting acquired data to the monitoring and controlling center for processing.

A general overview of the proposed intelligent traffic system is shown in Table 1. The structure of the proposed traffic IoT system consists of three layers: application, network and acquisition.

Main functions of the application layer are collecting, storing, and processing traffic data to produce value-added services; presenting the interface of traffic IoT to users and analyzing received information from acquisition layer according to the different needs.

The application layer includes the following subsystems:

- Intelligent Driver Management Subsystem: drivers can acquire real-time traffic information with minimum delay.

- Vehicle Guidance and Road Information Management Subsystem: monitoring number of vehicle on one road, tracking vehicle's violation, sending warning massages, guide drivers to avoid possible crowded sections based on the prediction of the traffic network, real-time traffic navigation, etc.

- Intelligent Traffic Management Subsystem: the traffic system database contains data from vehicle sensors, weather information from environmental sensors, and information on traffic flows. The subsystem processes received information and shares it through the interface with other subsystems. It allows tracing the location of a vehicle fast and accurate and optimizing traffic scheduling.
TABLE I. INTELLIGENT TRAFFIC IOT

\begin{tabular}{|c|c|c|c|c|}
\hline $\begin{array}{c}\text { Application } \\
\text { Layer }\end{array}$ & $\begin{array}{c}\text { Intelligent } \\
\text { Traffic } \\
\text { Management }\end{array}$ & $\begin{array}{c}\text { Intelligent } \\
\text { Driver } \\
\text { Management }\end{array}$ & $\begin{array}{c}\text { Information } \\
\text { Collection \& } \\
\text { Monitoring }\end{array}$ & $\begin{array}{c}\text { Information } \\
\text { Services }\end{array}$ \\
\hline $\begin{array}{c}\text { Network } \\
\text { Layer }\end{array}$ & Internet & WiFi, 3G/4G & WiMax & GPS, GPRS \\
\hline $\begin{array}{c}\text { Acquisition } \\
\text { Layer }\end{array}$ & RFID & RFID Reader & WSN & $\begin{array}{c}\text { Intelligent } \\
\text { Terminals }\end{array}$ \\
\hline
\end{tabular}

- Information Collection And Monitoring Subsystem: real-time distribution the information of road conditions, weather information, accident monitoring, etc. The subsystem merges data from different subsystems and provides it to end users in a suitable format.

- Information Service Subsystem: performs online vehicle information query and dynamic statistic analysis of real-time traffic flow, tracks a specific vehicle and generates reports for traffic management department.

The network layer, also called transport layer, is constituted by all sorts of private networks, Internet, wired and wireless communication networks, network management system, global positioning system(GPS), wireless general packet radio service (GPRS), worldwide interoperability for microwave access (WiMax), wireless fidelity (WiFi), Ethernet, and corporate private networks. It is responsible for transmitting data with high reliability and security, and processing the information coming from acquisition layer. GPRS provides high-speed wireless IP services for mobile users and fully supports the TCP/IP. The wireless communication channels used by the devices may include any of the prevailing standards such as IEEE 802.11, Zigbee or Bluetooth, etc.

Acquisition layer is constituted by all kinds of sensors and sensor gateways such as RFID, WSN, cameras, intelligent terminals to transmit data of mobile objects and other sensors used to collect real-time traffic and object identification information. It serves as a source of all types of information (for example, identified objects, traffic flow, etc.) collected from the physical world. Its main functions are to collect realtime information from IoT sensors, monitor objects and transfer data to the network layer.

The system utilizes wireless sensors to obtain real-time traffic information, such as traffic condition on each road, number of vehicles, average speed, and so forth. Utilization of wireless sensors is very appropriate due to their low power consumption, low cost, distributed processing and selforganization. In order to achieve large-scale network layout the system uses wireless cluster sensor network. Each cluster has a set of wireless sensors and each set is represented by the head node. Data at the head nodes are delivered to the backend system by a mobile agent. 
Already some new vehicles are equipped with GPS and sensors capable of receiving and sending driving information to the monitor and control center via the satellite communication facilities at any time. GPS could be connected with the wireless sensor networks which can be used for measuring speed, driving direction.

\section{DEVELOPMENT OF AN AGENT-BASED INTELLIGENT TRAFFIC INFORMATION SYSTEM}

There are a large number of heterogonous devices within the traffic monitoring system using IoT. Among challenges of full deployment IoT is making complete interoperability of these heterogeneous interconnected devices which require adaptation and autonomous behavior. The major issue in IoT is the interoperability between different standards, data formats, heterogeneous hardware, protocols, resources types, software and database systems $[35,36]$. Another issue is necessity of an intelligent interface and access to various services and applications. It seems that mobile agents are a convenient tool to handle these issues, provide means for communication among such devices and handle the IoT interoperability. Adding to that mobile agent is a perfect choice in cases of disconnection or low bandwidth, passing messages across networks to undefined destination and to handle the interoperability of IoT. All messaging exchanges among agents are established via the TCP/IP Protocol.

A software agent is an autonomous executable entity that observes and acts upon an environment and acts to achieve predefined goals. Agents can travel among networked devices carrying their data and execution states, and must be able to communicate with other agents or human users. A multi-agent system is a collection of such entities, collaborating among themselves with some degree of independence or autonomy.

Applying agent technology in the process of monitoring and control traffic is new approach. Such technology perfectly fits for distributed and dislocated systems like traffic monitoring and controlling due to its autonomy, flexibility, configurability and scalability thus reducing the network load and overcoming network latency. Agents can also be used to pass messages across networks where the address of destination traffic device is unidentified. Each traffic object is represented as a software agent (an intelligent object agent). In this infrastructure the extremely large variety of devices will get interconnected, and will be represented by its own intelligent agent that collects information and responds to others' requests. Agents will provide their functionality as a service. Autonomous intelligent agents are deployed to provide services necessary for the execution of functional tasks in each layer of the proposed architecture.

An agent is embedded within each device and each device supports all agent functions such as migration, execution. Whole system can be controlled by the specific application written for each device's mobile agent defining how it should behave and act intelligently. Mobile agents within the network migrate from one node to another allowing the devices to pass information to others, retrieve information and discover available resources.

Main IoT Traffic agents are:
- Traffic Mobile Agent: Transmits/receives different types of information to/from other objects the Internet; interprets the data coming from other objects (RFID, sensors, users), and provides a unified view of the context; communicates with other agents in the network to accomplish a specific task. All messages sent from this agent will be transferred to the traffic management system and communicate directly with a static agent of the intended application of the traffic management system mentioned above.

- User Agent: provides users with real-time information of entities residing in the system. The user agent is a static agent that interacts with the user. It is expected to coordinate with mobile agents.

- Monitor Agent: monitors the system to detect contingency situations and triggers some actions to react to some tag reading events on behalf of a smart traffic object, for example in emergency cases.

- RFID Agent: responsible for reading or writing RFID tags. When reading a tag, according to the data retrieved from it, this agent performs appropriate operations in handling a single task on behalf of a smart object of the associated RFID and to migrate to different platforms at run time.

- Sensor Agent: receives, processes data that have been read from the associated sensor and saves (or send it somewhere).

- Traffic Light Agent: detects irregular traffic conditions and changes the traffic control instructions right away.

- Camera Agent: is responsible for image collecting. All communications between camera agent and video Web server are conducted via the network layer. Camera agent can takes advantage of the existing infrastructure of the camera-based traffic monitoring systems that already available in many cities.

The traditional traffic monitoring system based on imageprocessing technology has many limitations. One of them is the impact of the weather. In case of thick dust, heavy rain, etc., the license plate cannot be seen clearly, so its image cannot be captured. The development of e-plate based on RFID provides a good opportunity for intelligent traffic monitoring and vehicle's identification and tracking [37]. If no agents are associated with the RFID tags (identification-centric RFID systems), then they may function as an independent set of programs for tag processing and communicate using standardized software agent protocols. The author suggests utilizing the agent technology within the e-plate based on RFID and other traffic objects to fully realize the combined potential of RFID and software agent technology.

An RFID-based smart traffic object (code-centric RFID systems) requires a substantial amount of memory space to store traffic object logics and data. The code-centric RFID systems can be used to store a mobile agent into the RFID tags that will enable integration with other parts of the traffic system. Using such technology in the Traffic Information System will eliminate the need for searching of the associated 
RFID-code information from a database and reduce overall system response time by retrieving service information from the tags [38], thus achieve faster service responses and perform on-demand actions for different objects in different situations. Each smart vehicle's RFID object consists of two components, namely, object processing logics and object data [39]. The object data contains a global unique Electronic Product Code (EPC) code as its unique identifier. Each RFID-tagged traffic object may be assigned an IPv6 Mapped EPC address [40]. The IoT networks are expected to include billions of devices, and each shall be uniquely identified. A solution to this problem is offered by the IPv6, which provides a larger address space of 128-bit address field to accommodate the increasing number of devices in IoT, thus making it possible to assign a unique IPv6 address to any possible device in the IoT network.

RFID can be used as a transponder in vehicle registration plate equipped with a RFID tag and sensors so that each car can get data it needs from the spot and deliver to assigned destination. The vehicle RFID tag stores information on the vehicle and its owner, such as plate number, vehicle type, speed, time when the car reaches the monitoring point, driver's name and license number. It can be used to estimate the number of vehicles in the road, average speed of vehicles, vehicle density, etc. The data from each vehicle is captured by fixed or mobile RFID reader at a monitoring station as information of the vehicle and will be sent to central server unit for collecting, processing and storing. Once system connects to the internet, all information of vehicles on each road segment is immediately saved in database and can be used for any purpose and application (vehicle tracking, monitoring or traffic information, etc.).

When a vehicle with an RFID tag passes through each monitoring station along the road, the RFID reader at those points will automatically read the tag data related to the vehicle and its owner and transmit to the wireless sensor active nodes. These nodes send accumulated data to the cluster head node. At the same time, a GPS receiver installed at the monitoring station can communicate with GPS satellites to obtain its position information that is taken as a position parameter of the vehicle. Then the data is transmitted using GPRS scheme to the real-time central database where the data is constantly updated to ensure data reliability.

\section{TRAFFIC SIMULATION FRAMEWORK}

To justify the proposed system online distributed traffic simulation was conducted. Simulation allows us to observe the properties, characteristics and behaviors of the traffic system. Based on detailed real-time data collected from the distributed online simulations, the IoT traffic system can provide accurate information necessary for near real-time traffic decisions.

The whole traffic IoT network is partitioned into dynamic overlapped sections, and a simulation processor is mapped to each section. Each simulation will be supplied with real-time data from nearby RFIDs and sensors and enabled to run continuously. The overall distributed simulation consists of a collection of such segment simulations where each small segment of the overall traffic IoT network is modeled based on local criteria. Each simulation segment is operating in an asynchronous mode, meaning each simulator executes independently of other simulators and the simulation server.

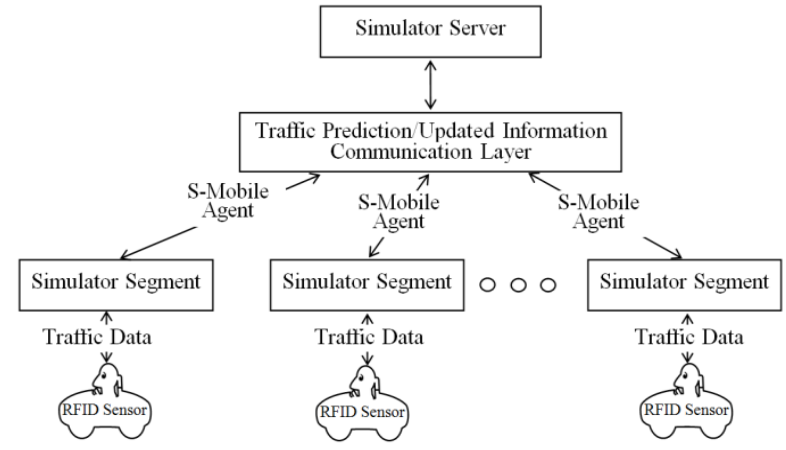

Fig. 2. Distributed online traffic simulation framework

These simulation segments are allowed to exchange information on vehicles moving from one simulation segment to another. Each simulator's segment locally models current traffic conditions and concentrating only on its area of concern. A simulator's segment, for example, might model some set of roads and intersections of that segment, and predict the rates of vehicle flow on links carrying vehicles out of that segment. Each segment shares its predictions with other simulation segments to create an aggregated view of both the individual segment's area of interest and the overall of traffic system. Simulators' segments publish their current traffic state information (speed, travel time, flow rate, etc.) and their predictions to the simulation server. An aggregation of all simulation segments provides an accurate estimation of a future state of the system.

The general model of distributed traffic simulation framework described in fig. 2. The simulation server disseminates information among the simulator segments, coordinates all simulators' segments and provides a predictive model of traffic conditions in specified traffic areas by analyzing and integrating the results of distributed simulators of those areas. The simulation server maintains state information of current and future operations of the traffic network such as flow rates, average speed, and the time when that information was generated. Running online simulations are integrated with traffic information system infrastructure to receive real-time traffic data and this overall simulation provides detailed information required for prediction of the system future states of the system. Detailed traffic information (such as speed, location, average acceleration of vehicles on the network segment and the current state of traffic control devices) generated during simulation is saved and managed on the simulation server.

Online distributed traffic simulation is a powerful approach for analyzing the characteristics and behavior of the traffic system and determining traffic conditions and help to reduce vehicle delay time of on the road, traffic congestion without the need of making costly changes in real world; prevent dangerous situations and delays by broadcasting messages informing drivers in the area to avoid congested roads [41]. It will be beneficial to transportation management as well as urban planning and architecture working on enhancement of 
the roads capacity, building new roads or to improve the existing roads and improvement of public transportation systems.

The current large-scale distributed simulation methodologies require tremendous network bandwidth and huge amount of computation by each simulator host. Mobile agents are used to reduce the communications loads placed in the network. Agents communicate with a specific simulation segment, providing all of the state information that was sent to the simulator server.

NetLogo simulator has been used for modeling a collection of adjacent intersections. Static and mobile agents represent different features of the network. Motor vehicles have been modeled individually within NetLogo using mobile agents. Simulation can be run on several computers. NetLogo allows giving instructions to large number of independent agents which could all operate at the same time. In this cause the NetLogo model runs in a single machine computing environment, but it can be extended to run on cluster of computers.

Four types of agents used in NetLogo: patches are used to represent static agent, turtles for mobile agent; links are used to make connections between turtles; and the observer for observing everything going on in the simulated environment [42]. The environment of the NetLogo is written entirely in Java, therefore patches and turtles are programmable by the user of NetLogo in Java language. In this simulation, the agent entities are vehicle, traffic lights, and sensors of intersections and lanes. Agents are created and randomly distributed over the network of intersections. A random number of vehicles were generated according to limits defined in the model. Sensors obtained the number of passing vehicles. The traffic lights action are based on goals of minimizing the waiting time of vehicles travelling through intersections and increasing throughput of vehicles that successfully pass through these intersections.

During each run, the following indicators were produced: not moving vehicles, average waiting time and average of speed of the vehicles in a time step. The human factors play an important role in traffic systems. In most cases the driver's behavior are unpredictable. Modeling of drivers' behavior using agent-based has been performed based on techniques proposed by [43].

The simulation has 'setup' and 'go' buttons. The 'setup' button calls a procedure to reset the model to the initialization state, and the 'go' button calls a procedure that carries out all actions for each simulation run. All visual aspects are managed by the NetLogo simulation, and after every run visualizations are automatically updated. The interface and performance evaluation of the simulation results are shown in fig. 3 .

\section{CONCLUSIONS AND FUTURE RESEARCH}

This paper presents a real-time traffic information collection and monitoring system architecture to solve the problem of real-time monitoring and controlling road vehicles.

The proposed architecture employs key technologies: Internet of Things, RFID, wireless sensor network (WSN),
GPS, cloud computing, agent and other advanced technologies to collect, store, manage and supervise traffic information.

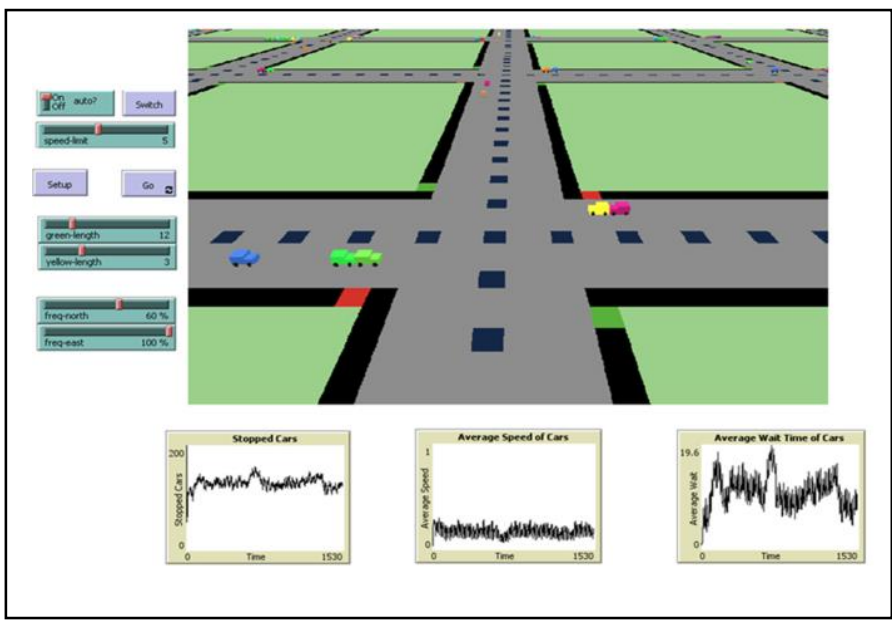

Fig. 3. Interface and performance evaluation of the simulation results

Agents provide an effective mechanism for communication amongst networked heterogeneous devices within the traffic information system.

The proposed system can provide a new way of monitoring traffic flow that helps to improve traffic conditions and resource utilization. In addition, transport administration department, using real-time traffic monitoring information, can in time detect potentially dangerous situations and take necessary actions to prevent traffic congestion and minimize number of accidents thus ensuring safety of road traffic. In general, the IoT will play an important role in the traffic management enhancing the efficiency of information transmission, improving traffic conditions and management efficiency, traffic safety, and reducing management costs.

However, the proposed traffic system based on the IoT consists of a large number of RFIDs and sensors that transmit data wirelessly. This calls for improved security to protect such massive amounts of data and privacy of users. It's a challenge for future research to ensure the security of smart objects in the traffic monitoring management system in case of a cyberattack or an intentional interest to a member of the IoT infrastructure. IoT requires modification of network connectivity models and readiness for massive increase in amount of real-time information. To achieve that, interaction communication models must be redesigned to include machine to machine and people to machine communications. Another research area is processing and analytics of large volumes of disparate data from Traffic IoT system to create applications that improve the flow of vehicles throughout the city.

\section{REFERENCES}

[1] Laisheng Xiao, "Internet of Things: a New Application for Intelligent Traffic Monitoring System”, Journal of Networks, 2011, vol. 6, No. 6.

[2] J. R.Molina, J. F. Martínez, P. Castillejo and L. López, “Combining Wireless Sensor Networks and Semantic Middleware for an Internet of Things-Based Sportsman/Woman Monitoring Application”, Sensors, 2013, vol. 13, pp. 1787-1835. 
[3] European Lighthouse Integrated Project - 7th Framework, Internet of Things - Architecture. http://www.iot-a.eu/, 2012.

[4] K. Kotis, \& A. Katasonov, "Semantic Interoperability on the Web of Things: The Smart Gateway Framework", In Proceedings of the Sixth International Conference on Complex, Intelligent, and Software Intensive Systems (CISIS-2012), Palermo, 2012.

[5] A. J. Jara, S. Varakliotis, A. F. Skarmeta and P. Kirstein, "Extending the Internet of Things to the Future Internet through IPv6 support", Mobile Information Systems, IOS Press, 2013.

[6] Laura Jarvenpaa, et al, "Mobile Agents for the Internet of Things", 17th International Conference on System Theory, Control and Computing (ICSTCC), 2013.

[7] Leppänen, Teemu, Liu, Meirong, et al., "Mobile Agents for Integration of Internet of Things and Wireless Sensor Networks", 2013 IEEE International Conference on Systems, Man, and Cybernetics.

[8] L. Atzori, A. Iera and G. Morabito, "The internet of things: A survey", Comput. Netw, 2010, vol. 54, no. 15, pp. 2787-2805.

[9] K. Ashton, "That Internet of Things thing”, RFiD Journal, 2009.

[10] http://newsroom.cisco.com/release/1308288, 2014.

[11] D. Bandyopadhyay and J. Sen, "The internet of things - applications and challenges in technology and Standardization", Springer International Journal of Wireless Personal Communications, 2011, vol. 58, no. 1, pp. 49-69.

[12] P. Guillemin and P. Friess, "Internet of things strategic research roadmap", The Cluster of European Research Projects, Tech. Rep., September 2009, http://www.internet-of-things-research.eu/pdf/IoT Cluster Strategic Research Agenda 2009.pdf [Accessed on: 2011-08-15].

[13] D. Singh, G. Tripathi and A. J. Jara, "A survey of Internet-of-Things: Future Vision, Architecture, Challenges and Services", IEEE World of Forum on Internet of Things.

[14] B. Xu, "Key IOT Technology and Application Research", Applied Mechanics and Materials, 2014, vol. 543-547, pp. 3411-3414.

[15] M. Pang and X. Zhao, "Traffic Flow Prediction of Chaos Time Series by Using Subtractive Clustering for Fuzzy Neural Network Modelling," Proceedings 2nd International Symposium Information Technology Application, Washington - DC, 2008, pp. 23-27.

[16] S.Bhadra, A. Kundu and S. K. Guha, "An Agent based Efficient Traffic Framework using Fuzzy”, Fourth International Conference on Advanced Computing \& Communication Technologies, 2014.

[17] V.Katiyar, P. Kumar and N. Chand, "An Intelligent Transportation System Architecture using Wireless Sensor Network", International Journal Computer Applications, 2011,vol. 14, pp. 22-26.

[18] P. Spiess, S. Karnouskos, D. Guinard, D. Savio, O. Baecker, L. Souza, et al., "SOA-based integration of the internet of things in enterprise services", In: Proceedings of IEEE ICWS 2009, Los Angeles, pp. 1-8.

[19] Libelium Communications Distribuend as S.L., "50 sensor applications for a smarter world", 2014, available at http://www.libelium.com/top 50 iot sensor applications ranking/.

[20] D. Miorandi, S. Sicari, F. De Pellegrini and I. Chlamtac, "Internet of things: Vision, applications and research challenges," Ad Hoc Networks, 2012, vol. 10, no. 7, pp. 1497-1516.

[21] M. C. Domingo, "An overview of the internet of things for people with disabilities," Journal of Network and Computer Applications, 2012, vol. 35 , no. 2 , pp. 584-596.

[22] T.S. Lo'pez, D.C. Ranasinghe and M. H. Duncan McFarlane, "Adding sense to the Internet of Things An architecture framework for Smart Object systems", Personal Ubiquitous Computing, 2012, vol. 16, pp. 291-308.

[23] P. Pyykonen, J. Laitinen, J. Viitanen, P. Eloranta and Korhonen, "IoT for Intelligent Traffic System, IoT for intelligent traffic system", International Conference on Intelligent Computer Communication and Processing (ICCP), 2013 IEEE.

[24] C.Yulian, L. Wenfeng and J. Zhang, "Real-Time Traffic Information Collecting and, Monitoring System Based on the Internet of Things", 6th International Conference on Pervasive Computing and Applications (ICPCA), 2011 IEEE.
[25] X. Yu, F. Sun and X. Cheng, "Intelligent Urban Traffic Management System Based on Cloud Computing and Internet of Things", International Conference on Computer Science \& Service System, 2012 IEEE, pp. $2169-2172$.

[26] Y. Yin and J. Dalin, "Research and Application on Intelligent Parking Solution Based on Internet of Things", 5th International Conference on Intelligent Human-Machine Systems and Cybernetics (IHMSC), 2013, pp. $101-105$.

[27] C. Goumopoulos and A. Kameas, "Smart Objects as Components of UbiComp Applications", International Journal of Multimedia and Ubiquitous Engineering, vol. 4, no. 3.

[28] F. Kawsar, "A Document based Framework for User Centric Smart Object Systems", Ph.D. Dissertation, Waseda University, Tokyo.

[29] F. Kawsar and T. Nakajima, "A Document Centric Framework for Building Distributed Smart Object Systems", in 2009 IEEE International Symposium on ObjectiComponentiService-Oriented Real-Time Distributed Computing, Tokyo, 17-20 March 2009, pp. 71-79.

[30] G. Nakamiti, V.E. Silva and J.H. Ventura, "An Agent-Based Simulation System for Traffic Control in the Brazilian Intelligent Cities Project Context”, Proc. 2012 Agent Direct Simulation Conference, Orlando FL.

[31] B.Chen, H.H. Cheng, and J. Palen, "Integrating mobile agent technology with multi agent systems for distributed traffic detection and management systems", Transport Research, 2009, vol.17, no. 1, pp. 110.

[32] T. Karthikeyan and S.Sujatha, "Optimization of Traffic System using TCL Algorithm through FMSA and IMAC Agents", International Journal of Advanced Research in Computer Engineering \& Technology (IJARCET), 2012, vol. 1, no 6.

[33] G.Fortino, A. Guerrieri, W. Russo and Cl. Savaglio, "Integration of Agent-based and Cloud Computing for the Smart Objects-oriented IoT", Proceedings of the 18th International Conference on Computer Supported Cooperative Work in Design, 2014 IEEE.

[34] W. W. Godfrey, S. S. Jha and B. N.Shivashankar, "On A Mobile Agent Framework for an Internet of Things", 2013 International Conference on Communication Systems and Network Technologies.

[35] A. Katasonov, O. Kaykova, et al.,"Smart Semantic Middleware for the Internet of Things," In: Proceedings of the 5th International Conference on Informatics in Control, Automation and Robotics, Intelligent Control Systems and Optimization, 2008, pp. 169-178.

[36] T.Leppanen, L.Meirong, et al., "Mobile Agents for Integration of Internet of Things and Wireless Sensor Networks", 2013 IEEE International Conference on Systems, Man, and Cybernetics (SMC), pp. $14-21$.

[37] Evizal,T. Abd Rahman, S. K. Abdul Rahim, "RFID Vehicle Plate Number (e-Plate) for Tracking and Management System", 2013 EEE International Conference on Parallel and Distributed Systems, pp: 611 616.

[38] M. Chen, S. González, Q. Zhang and V. C.M. Leung, "Code-Centric RFID System Based on Software Agent Intelligence", IEEE Intelligent Systems, 2010.

[39] M. Chen, S. Gonzalez-Valenzuela, Q. Zhang and V. Leung, "Software agent-based intelligence for code-centric RFID Systems," IEEE Intelligent Systems, 2010, vol. 99.

[40] M. C. Chung, G. M. Lee, N. Crespi and C. C. Tseng, "RFID Object Tracking with IP Compatibility for the Internet of Things", 2012 IEEE International Conference on Green Computing and Communications, Conference on Internet of Things, and Conference on Cyber, Physical and Social Computing.

[41] W. Suh, M. P. Hunter and Richard Fujimoto, "Ad hoc distributed simulation for transportation system monitoring and near-term prediction", Journal of Simulation Modelling Practice and Theory, 2014, vol. 41, pp. 1-14.

[42] U. Wilensky, NetLogo User Manual version 4.1.3, Center for Connected Learning and Computer-Based Modelling, Northwestern University, Evanston, IL. Available at: http://ccl.northwestern.edu/netlogo/. As accessed on July 2014.

[43] R. J. F. Rossetti, R. H. Bordini and A. L. C. Bazzan, "Using BDI agents to improve driver modelling in a commuter scenario", Transportation Research, Part C, pp. 373-398, 2002. 